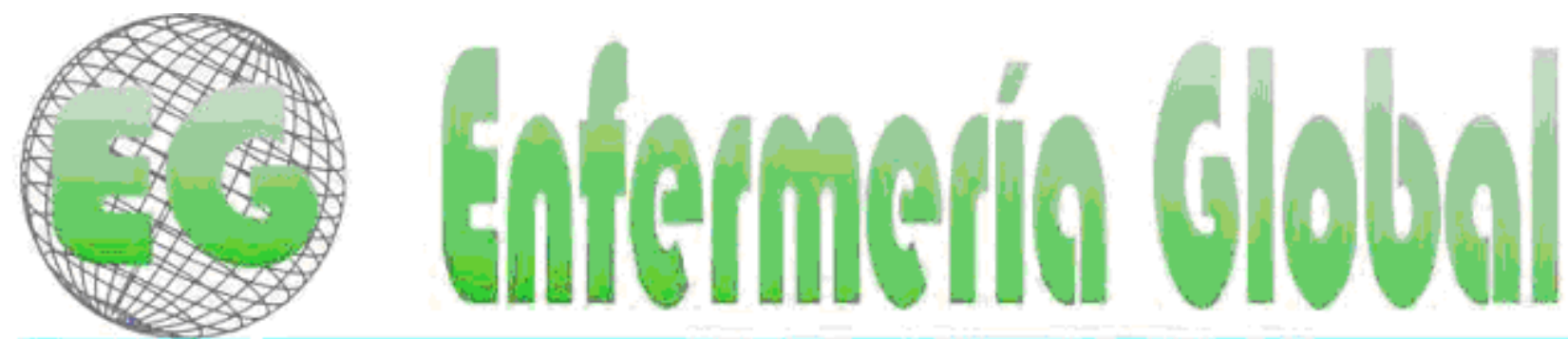

15SN 1696-6*5:

Revista electrônica cuatrimestral de Enfermeria

$\mathrm{N}^{\circ} 20$

Octubre 2010

www.um.es/egloball

CLÍNIGA

\title{
PERFIL BIOPSICOSOCIAL DE LA MADRE Y SU RELACIÓN CON EL ABANDONO DE LACTANCIA MATERNA EXCLUSIVA
}

BIO-PSYCHOSOCIAL PROFILE OF THE MOTHER AND HER RELATION WITH THE ABANDONMENT OF EXCLUSIVE MATERNAL LACTATION

\author{
*Cardenas, MH., Montes, E, Varon, M., Arenas, N., Reina, R. \\ *Universidad de Carabobo, Facultad de Ciencias de la Salud, Escuela de Enfermería. Venezuela. \\ Palabras Clave: Perfil biopscosocial, destete, lactancia materna, crecimiento y desarrollo \\ Keywords: Bio-Psychosocial Profile, Weaning, Maternal Lactation, Growth, and Development
}

\section{RESUMEN}

La lactancia materna exclusiva es una forma inigualable de nutrición infantil. Sin embargo, a pesar de la importancia que tiene la lactancia materna exclusiva, en la actualidad existe un problema de abandono precoz de la misma debido a la falta de políticas efectivas en pro de su promoción. El objetivo de esta investigación fue establecer si existe relación entre el perfil biopsicosocial de la made y el abandono de la lactancia materna exclusiva. La metodología fue enmarcada dentro del diseño cuantitativo, de tipo correlacional de corte transversal. La población fue de 106 madres de niños menores de seis meses que asistían a las consultas de niños sanos de la unidad sanitaria Dr. Cándido Díaz Carballo y el Grupo Médico Miranda, la muestra fue representativa del Universo conformada por 60 madres, distribuida en dos grupos: 30 madres que daban lactancia materna exclusiva y 30 que habían abandonado la misma. Los datos se presentan en cuadros de distribución de porcentajes utilizando el estadístico de chi cuadrado con corrección de Yates. Resultados: la edad, el aspecto psicológico, las costumbres de la madre guardan relación estadísticamente significativa con el abandono de la lactancia materna exclusiva. Se concluye que los factores biopsicosociales como son la edad, la paridad, el aspecto psicológico, la cultura se puede convertir en obstáculo para que la madre practique una lactancia materna exclusiva y el aspecto social como es el status social no tiene relevancia para el abandono de lactancia materna exclusiva al igual que la procedencia de la madre 


\section{ABSTRACT}

Exclusive maternal lactation is a form of infant nutrition without equal. Nevertheless, in spite of the importance of exclusive maternal lactation; at the present time a problem of its early abandonment exists due to the lack of effective policies for its promotion. The objective of this investigation was to establish if a relation between the bio-psychosocial profile of the mother and the abandonment of exclusive maternal lactation exists. The methodology was framed within the quantitative design, of cross section correlation type. The population was 106 mothers of children under the age of six months that visited the pediatrics department of the health unit Dr. Diaz Carballo and the Medical Group Miranda, the sample was representative of the whole conformed by 60 mothers, distributed in two groups: 30 mothers who gave exclusive maternal lactation and 30 that had stopped. Data appear in graphs of percentages using statistics of the square of chi with correction of Yates. Results: the age, the psychological aspect, the habits of the mother maintain a statistically significant relation with the abandonment of exclusive maternal lactation. One concludes that the bio-psychosocial factors (age, parity, psychological aspect, and culture) can become obstacles to the mother practicing exclusive a maternal lactation. The social aspect (social status) does not have relevance to the abandonment of exclusive maternal lactation, similarly to the origin of the mother.

\section{INTRODUCCIÓN}

La lactancia materna es el estándar dorado de alimentación del niño; es el arte de ser madre, pero debe aprenderse ya que esto resulta algo nuevo para las madres (1). En el Estado Cojedes, específicamente en el municipio San Carlos, existen diez ambulatorios urbanos que ofrecen el programa de control de niños sanos y ocho consultorios privados que igualmente brindan el mismo servicio; en estos programas se observa la poca motivación en las madres para amamantar a sus hijos, alegando una serie de razones tales como: el niño no queda satisfecho, llora mucho, la leche no es buena, el tamaño de los senos no es el adecuado, entre otras; a pesar que en el Estado existe una liga defensora de la lactancia materna dirigida por la Fundación del Niño, con el fin de convertirse en un estado promotor de la lactancia materna exclusiva; la liga está integrada por un equipo multidisciplinario que tiene como función la propagación e información referida a la lactancia materna exclusiva.

En la unidad sanitaria Dr. Díaz Carballo y el grupo Médico Miranda se atienden madres de diferentes estratos sociales, nivel económico y educativo, que provienen tanto de la zona urbana como rural, en ellas se observa el rechazo o la ambivalencia que tienen hacia la lactancia materna exclusiva; tomando en cuenta experiencias previas se cita un estudio correlacional realizado en Venezuela (Estado Cojedes), programa educativo sobre la lactancia materna exclusiva dirigido a las adolescentes que asisten a la consulta prenatal del ambulatorio Menca de Leoni se pudo concluir que las adolescentes no poseen el conocimiento sobre la importancia de la lactancia materna y las técnicas de amamantamiento (2).

En otro estudio cuasiexperimental titulado nivel de conocimiento del grupo familiar de las adolescentes embarazadas sobre la lactancia materna exclusiva, con el objetivo de comparar el nivel de conocimientos del grupo familiar de la adolescente sobre ventajas y técnicas de la lactancia materna exclusiva antes y después de participar en un programa educativo, se concluyó que el nivel de conocimientos de los grupos familiares se incrementó después de participar en el programa educativo (3).

La lactancia materna es un fenómeno biocultural por excelencia en los humanos ya que además de biológico es modificado por la cultura, se dice que es la nutrición óptima para el crecimiento y desarrollo de los niños (4). Asimismo la lactancia materna ofrece ventajas al niño y a la madre, las mismas se clasifican de la siguiente manera: para el niño, favorece el 
crecimiento y desarrollo si es amamantado exclusivamente hasta los seis meses y luego complementando correctamente con papillas y sólidos hasta los dos años, aporta los nutrientes específicos para el sistema nervioso central, sus patrones de crecimiento y desarrollo sensorial y emocional son mas armónicos, tienen mayor coeficiente intelectual y mayor desarrollo psicomotor, padecen de un menor numero de episodios de diarrea, las infecciones respiratorias también son mas leves ya que esta leche aporta componentes inmunes al sistema inmunológico del niño. Definitivamente la lactancia materna exclusiva protege al niño de patologías infecciosas, metabólicas, carenciales, gastrointestinales, odontológicas, muerte súbita, abandono y maltrato (5).

Las ventajas para la madre son las siguientes: mejora su autoestima, logra un vinculo afectivo hacia su hijo, controla su ciclo reproductivo, ejerce un derecho, manifiesta sentimientos positivos de satisfacción personal y emocional, le gratifica criar un bebé más sano, logra recuperarse física y emocionalmente después del parto, contribuye a recuperar la silueta y a espaciar los embarazos, protege de patologías (sangrados, anemias, cáncer de mamas, útero y ovario). En relación a lo antes expuesto cabe mencionar la importancia que tiene el control prenatal para promover la lactancia materna exclusiva, ya que es en este periodo cuando se hace posible educar e informar a las embarazadas sobre todo lo concerniente a la lactancia materna; de igual manera se pueden identificar factores que incidan en el abandono precoz de la misma.

Por lo tanto es necesario precisar las causas que obstaculicen la lactancia materna, enseñar sobre los beneficios de la lactancia para la madre y el niño, orientar sobre técnicas y desarrollo de los pezones, entrenar sobre técnica de amamantamiento, dar a conocer a la madre que trabaja sus derechos legales, realización de demostraciones sobre técnica de extracción de leche y posterior utilización (6). La edad y la paridad forman parte del perfil biológico de la madre, es por ello que se destaca que la edad materna influye en la práctica de la lactancia materna exclusiva, por ejemplo, las madres adolescentes tienen más probabilidad de abandonar la lactancia materna por influencia de su grupo familiar (3). Sobre este particular es probable que se deba al desconocimiento por parte de la familia sobre los beneficios de la lactancia materna.

Por otra parte "las mujeres de mayor edad fisiológicamente sufren un retardo en el ciclo de oxitoxina-prolactina y debido a esto tardan más en obtener una producción completa de leche" (7). Sin embargo con una buena orientación y apoyo estas madres pudieran superar este obstáculo sin necesidad de acudir a la administración de sucedáneos. La paridad también podrá influir en el abandono de la lactancia materna cuando se reporta que el número de hijos consigue ser de ayuda al éxito o fracaso durante el amamantamiento (7).

Existen además los factores psicológicos que pudieran estar influyendo en el destete precoz de las madres, esto puede deberse aque el estado emocional de la madre repercute en la lactancia materna ya que las situaciones de estrés, preocupación, depresión, entre otras, disminuyen fisiológicamente la producción de leche (8). Sobre este tema se dice que los estados de ánimo negativos inhiben la secreción de oxitocina, sustancia primordial para la eyección de la leche, es por eso que la madre debe conocer cómo influye el estado emocional en la producción y eyección de leche.

Los factores sociales juegan un papel importante en el deterioro de la lactancia materna, estos pueden ser modificaciones en la estructura social sobre todo a nivel familiar, la entrada de la mujer al campo de trabajo, la era de la industrialización que tiene que ver con el estrato económico y la migración de la zona rural hacia la ciudad (urbana); estos factores han 
influido a nivel mundial definitivamente en la casi erradicación o abandono de la lactancia materna exclusiva. (9).

Todos estos aspectos le ofrecen a la madre muchas opciones de alimentación para su hijo y es cuando ella decide abandonar la lactancia materna para introducir sucedáneos de la leche materna a la alimentación del niño como: las formulas lácteas a base de leche de animales de vaca y vegetales, la soya, papillas de cereales o harinas con leche artificial, compotas de frutas, o simplemente agua y así trasgreden la buena nutrición que pudieran lograr dando lactancia materna exclusiva hasta los seis meses de edad (6).

La escolaridad o grado de instrucción de la madre es importante tomarlo en cuenta cuando se le está educando sobre la lactancia, porque a mayor escolaridad tendrá mayor ventaja de entender las recomendaciones dadas sobre el tema: "una de las estrategias básicas para motivar y lograr que las madres alimenten a sus hijos al pecho es la educación. Las mujeres no amantan por instinto; el amamantamiento es una destreza que se debe aprender" (10). De ahí que la orientación y educación que la madre reciba en el control prenatal, sala de parto, puerperio o consultas de niños sanos sobre la lactancia materna será determinante para el amamantamiento exclusivo.

Mucha gente cree que la lactancia materna por ser un proceso natural es algo sencillo; pero esto no es totalmente cierto, ya que alrededor de la mitad de las mujeres tienen problemas para amantar a sus hijos, sobre todo si se trata de su primer hijo, de una madre sin apoyo familiar, especialmente si es adolescente (11).

La madre que amamanta está cumpliendo con uno de los roles maternos mas importantes como es la alimentación de su hijo, pues de esto depende su subsistencia. La adopción del rol maternal es un proceso interactivo y evolutivo que se produce durante el embarazo y proceso del parto, en el cual la madre se va sintiendo vinculada a su hijo, adquiere competencia en la realización de sus cuidados asociados a su rol y experimenta placer y gratificación dentro de dicho rol (12).

De acuerdo con la Teoría de Mercer el rol materno es algo que debe aprender la madre y va a depender de su entorno social, familiar, cultural; es por eso que todos estos factores afectarán de una manera u otra a la lactancia materna, exclusiva actividad principal del rol materno (12).

El objetivo del estudio fue establecer si existe relación entre el perfil biopsicosocial materno en su factor biológico, psicológico y social y el abandono de la lactancia materna exclusiva a nivel de una consulta de niños sanos. La relevancia de este estudio radica en que permitirá identificar el perfil de la madre que tiende a abandonar la lactancia materna exclusiva, así el personal de enfermería clasificará a la madre, de acuerdo al perfil y podrá saber cuál tiene más posibilidad de abandonar la lactancia materna exclusiva, pudiendo ser atendida con mayor prontitud e intensidad.

\section{MATERIAL Y MÉTODOS}

La investigación es un diseño cuantitativo de tipo correlacional de corte transversal. La población estuvo conformada por 106 madres que asistieron al control de niños sanos con hijos menores de seis meses de edad; en la Unidad Sanitaria Dr. Cándido Díaz Carballo y el Grupo Médico Miranda. La muestra seleccionada fue estadísticamente representativa del universo en estudio siendo de tipo probabilística y estratificada ya que hubo dos grupos de madres: las que daban lactancia materna exclusiva y las que abandonaron la lactancia materna exclusiva. Cada grupo estuvo conformado por 30 madres. Se le aplicó un 
instrumento estructurado en dos partes: la primera relacionada al status económico medidos por el método Graffar-Méndez (modificado por Méndez Castellano), la segunda parte está conformada por treinta y un ítems con I escala tipo liker, con cinco alternativas. El instrumento fue validado por expertos. Para la confiabilidad fue necesario aplicar una prueba piloto a diez elementos muestrales con características similares a los de la muestra objeto de estudio, los datos de la prueba fueron sometidos al estadístico Alfa de Cronbach obteniendo como resultado 0,76 siendo una confiabilidad aceptable lo que permitió aplicar el instrumentó a la muestra objeto de estudio.

La recolección de datos se hizo de la siguiente manera: se informó por escrito a los directivos de las instituciones sobre el objetivo del estudio, se solicitó consentimiento informado a las madres para que participaran en el estudio, se les explicó los objetivos del estudio a las madres y el procedimiento de la recolección de datos, posteriormente se aplicó el instrumento a la muestra seleccionada. Para la presentación e interpretación de los resultados se utilizó como estadístico las frecuencias absolutas y relativas y el Chi Cuadrado con corrección de Yates y por otra parte para las características psicológicas se utilizó el test de la $U$ de Mann-Whitney (16), es bueno aclarar que las características psicológicas y las costumbres se dividieron en positivas y negativas según sea el caso de acuerdo a la lactancia materna exclusiva; asimismo los resultados se presentaron en cuadros con su respectivo gráfico.

\section{RESULTADOS}

\section{CUADRO 1.}

Distribución de las madres según grupos de edad y frecuencia de abandono de la lactancia

\section{Lactancia materna exclusiva}

\begin{tabular}{lcc}
\hline EDAD (AÑOS) & $\mathrm{SI} \mathrm{F}(\%)^{*}$ & $\mathrm{NO} F(\%)^{*}$ \\
\hline $16-19$ & $5(16.7)$ & $13(43.3)$ \\
20 O MÁS & $25(83.3)$ & $17(56.7)$ \\
\hline TOTAL & $30(100.0)$ & $30(100.0)$ \\
\hline
\end{tabular}

*porcentajes en base al total de cada grupo

chi $^{2}$ (yates) $=3.889 ; \mathrm{g} . \mathrm{I} .=1 ; \mathrm{p}<0.05$. Asociación significativa

Fuente: instrumento aplicado.

Se determinó que en el grupo de madres que abandonaron la lactancia materna exclusiva predominó el grupo de 20 o más años con un 83.3\%, siendo las adolescentes en este grupo sólo el $16.7 \%$, mientras que en las madres que le dieron lactancia materna exclusiva el grupo de 20 años o más fue $56.7 \%$ y las adolescentes el 43.3\%. Se estableció una asociación significativa $(p<0.05)$ entre el abandono de la lactancia materna exclusiva y la edad según las categorías establecidas. Se puede inferir según los resultados que las madres adultas tienden abandonar con más frecuencia la lactancia materna exclusiva, esto se puede deber a que las mujeres de mayor edad tardan más tiempo para alcanzar la producción completa de leche, (14). 


\section{CUADRO 2}

Distribución de las madres según grupos de paridad y frecuencia de abandono de la lactancia

\section{Lactancia materna exclusiva}

\begin{tabular}{|c|c|c|}
\hline \multirow{3}{*}{$\begin{array}{l}\text { PARIDAD } \\
\text { PRIMÍPARAS } \\
\text { MULTIIPARAS }\end{array}$} & SI F (\%) ${ }^{*}$ & $\operatorname{NOF}(\%)^{*}$ \\
\hline & $8(26.7)$ & $11(36.7)$ \\
\hline & 22 (73.3) & 19 (63.3) \\
\hline TOTAL & $30(100.0)$ & $30(100.0)$ \\
\hline
\end{tabular}

*porcentajes en base al total de cada grupo

chi $^{2}$ (yates) $=0.308 ;$ g. I. $=1 ; p>0.05$. Asociación no significativa

Fuente: instrumento aplicado.

Se determinó que en el grupo de madres que abandonaron la lactancia materna exclusiva predominó el grupo multíparas con un $73.3 \%$, siendo las primíparas el $26.7 \%$, mientras que en las madres que le dieron lactancia materna exclusiva el grupo de multíparas fue de $63.3 \%$ y el de las primíparas $36.7 \%$. No se estableció una asociación significativa $(p>0.05)$ entre el abandono de la lactancia materna exclusiva y la paridad (número de hijos). Sin embargo, el número de hijos previos pudiera ser de gran ayuda dependiendo del éxito o fracaso obtenido durante el amamantamiento (14).

\section{CUADRO 3}

Distribución de las madres según respuesta consolidada de la ocurrencia positiva del factor psicológico en su indicador grado emocional

\begin{tabular}{lll} 
& $\begin{array}{l}\text {-actor psicológico } \\
\text { emocional }\end{array}$ & en su indicador gradc \\
\hline OCURRENCIA & SI $\mathrm{F}(\%)^{*}$ & $\mathrm{NO} \mathrm{F}(\%)^{*}$ \\
\hline NUNCA & $0(0.0)$ & $0(0.0)$ \\
CASI NUNCA & $1(3.3)$ & $0(0.0)$ \\
A VECES & $21(70.0)$ & $0(0.0)$ \\
CASI SIEMPRE & $8(26.7)$ & $29(96.7)$ \\
SIEMPRE & $0(0.0)$ & $1(3.3)$ \\
\hline TOTAL & $30(100.0)$ & $30(100.0)$ \\
\hline orcentajes en base al total de cada grupo u de mann whitney $=19.0 ; z=-6.404 ; p<0.05$.
\end{tabular}

Se determinó que en el grupo de madres que abandonaron la lactancia materna exclusiva predominó en la escala Graffar la clase II, sigue la clase V con $23.3 \%$, luego las clases III y IV con $16.7 \%$ cada una y con $10.0 \%$ la clase I el grupo de 20 o más años con un $83.3 \%$, siendo para el grupo de las madres que le dieron lactancia materna las más representadas la clases III y IV con $26.7 \%$, sigue la clase II con $23.3 \%$, sin nadie en la clase I ( $0.0 \%)$. A fin de poder aplicar el estadístico $\mathrm{CHI}^{2}$, se procedió a unir los cómputos de las clases I, II y II en una categoría y las clases IV y $\mathrm{V}$ en otra, con lo cual se estableció que no hubo una 
asociación significativa ( $p>0.05$ ) entre el abandono de la lactancia materna exclusiva y las clases sociales agrupadas según las categorías establecidas.

\section{CUADRO 4}

Distribución de las madres según respuesta consolidada de la ocurrencia del factor social en su indicador costumbres negativas por grupo de estudio

\section{Factor social en su indicador costumbres negativas}

\begin{tabular}{lll}
\hline OCURRENCIA & SI $F(\%)^{\star}$ & NO $F(\%)^{*}$ \\
\hline NUNCA & $0(0.0)$ & $30(100.0)$ \\
CASI NUNCA & $0(0.0)$ & $0(0.0)$ \\
A VECES & $6(20.0)$ & $0(0.0)$ \\
CASI SIEMPRE & $22(73.3)$ & $0(0.0)$ \\
SIEMPRE & $2(6.7)$ & $0(0.0)$ \\
\hline TOTAL & $30(100.0)$ & $30(100.0)$ \\
\hline
\end{tabular}

*porcentajes en base al total de cada grupo u de mann whitney $=0.000 ; z=-6.983 ; p$ $<0.05$. Distribuciones significativamente diferentes Fuente: instrumento aplicado.

Se determinó que en el grupo de madres que abandonaron la lactancia materna exclusiva predominó el grupo con procedencia urbana con un $70.0 \%$, siendo las que proceden del ambiente rural el $30.0 \%$, mientras que en las puérperas que le dieron lactancia materna exclusiva el grupo de madres fue igual la distribución $(50.0 \%)$ para la procedencia urbana o rural. No se estableció una asociación significativa $(p>0.05)$ entre el abandono de la lactancia materna exclusiva y la procedencia geográfica de las madres. Demostrándose que las madres del área urbana amamantan menos que las del área rural; esto pudiera deberse a que el factor social influye en el deterioro de la lactancia materna exclusiva debido a modificaciones en la estructura social, sobre todo, a nivel familiar, la entrada de la mujer al campo de trabajo, la era de la industrialización, etc.; a lo largo del tiempo estos factores han influido en la casi erradicación de la lactancia materna exclusiva a nivel mundial (14).

\section{DISCUSIÓN}

Según los resultados presentados, se puede decir que las madres adultas tienden a abandonar con más frecuencia la lactancia materna exclusiva, esto se podría deber a que las mujeres de mayor edad tardan más tiempo para alcanzar la producción completa de leche, y podrían tener experiencias de fracaso en cuanto al amamantamiento en embarazos anteriores (7), podría estar relacionado con el trabajo en el hogar (9) y carecen de apoyo familiar (12). Es importante señalar que estos resultados son contrarios a los encontrados por Evies en cuanto a que las madres adolescentes tienen mayor probabilidad de abandonar la lactancia materna exclusiva por influencia de su grupo familiar (3).

De acuerdo a lo representado anteriormente en los resultados se concluye que no se estableció una asociación significativa $(p>0.05)$ entre el abandono de la lactancia materna exclusiva y la paridad (número de hijos). Sin embargo Helsing afirma que el número de hijos previos pudieran ser de gran ayuda o no dependiendo del éxito o fracaso obtenido durante el amamantamiento (7). 
De acuerdo a las distribuciones de las respuestas consolidadas de ocurrencia del factor psicológico, en su indicador grado emocional difieren significativamente $(p<0.05)$, por lo que se puede asegurar con un error menor del $5 \%$ que las madres que abandonaron la lactancia materna exclusiva antes de los seis meses tienden a presentar una ocurrencia menor de las características psicológicas positivas interrogadas que las del grupo que no abandonan. Esto puede deberse a que las preocupaciones, temores, angustias, depresiones, interfieren negativamente en el proceso de amamantamiento. Se dice que el estado emocional de la madre repercute en la lactancia materna ya que las situaciones de estrés, preocupación, depresión entre otras disminuyen fisiológicamente la producción de leche (8).

Se estableció que las distribuciones de las respuestas consolidadas de ocurrencia del factor costumbres negativas difieren significativamente $(p<0.05)$, por lo que se puede asegurar con un error menor del $5 \%$ que las madres que abandonaron la lactancia materna exclusiva antes de los seis meses tienden a presentar casi siempre, en un 73,3\%, una ocurrencia mayor de las costumbres negativas como es darle sucedáneos a los niños antes de los seis meses, mientras que las madres que no abandonaron la lactancia materna exclusiva, el $100 \%$ manifestó no tener esas costumbres negativas. Al parecer muchas madres encuentran muy difícil de resistir y cada cual da una razón diferente, según sea el caso: el niño queda con hambre, hay que darles líquidos para quitarles la sed, el niño llora mucho, se me secó la leche, hay que acostumbrarlo para cuando no pueda amamantarlo, estas razones se deben a la costumbres o cultura de cada madre" (13). Y es ahí donde el personal de salud debe estar preparado para orientar a la madre durante y después del embarazo.

\section{CONCLUSIONES}

1.-Según los resultados obtenidos se pudo comprobar que la edad guarda relación estadísticamente significativa con el abandono de la lactancia materna exclusiva.

2.- En cuanto a la paridad y el abandono de la lactancia materna se pudo comprobar que no hay relación estadísticamente significativa.

3.- Por otra parte se pudo comprobar que el factor psicológico en su indicador grado emocional guarda relación con el abandono de la lactancia materna.

4.- El status social no guarda relación significativa con el abandono de la lactancia materna exclusiva.

5.- La procedencia de las madres no guarda relación con el abandono de la lactancia materna exclusiva.

6.- Asimismo las creencias guardan relación con el abandono de la lactancia materna exclusiva.

7.- De esta misma forma la hipótesis específica № 7 se comprueba ya que los resultados permiten inferir que las costumbres guardan relación con el abandono o el éxito de la lactancia materna exclusiva. (Ya sea negativa o positiva según sea el caso). 


\section{REFERENCIAS BIBLIOGRÁFICAS}

1. BUSTOS, María (2002), El núcleo Familiar y la Aceptación de la Lactancia Materna. Archivos Médicos de Familia. México.

2. PEREZ María et al: (2004), Programa Educativo Sobre la Lactancia Materna Exclusiva Dirigido a las Adolescentes que Asisten a la Consulta Prenatal del Ambulatorio Menca de Leoni. Trabajo Especial de Grado, Cojedes, Venezuela.

3. EVIES, Ani, (1998), Nivel de Conocimiento del Grupo Familiar de las Adolescentes Embarazadas Sobre la Lactancia Materna Exclusiva. Trabajo Para Optar a Titulo de Magíster, Carabobo, Venezuela.

4. BENAVIDES, Nelson. (2002), Principales Causas del Abandono de la Lactancia materna Exclusiva (amamantar), Revista de la Facultad de Salud Publica y Nutrición UANL Edición Especial № 2. Cuba.

5. OSORNO, Jairo, (1996), Hacia una Feliz Lactancia Materna, (Ventajas de la Lactancia Materna), Apoyo de la Lactancia Materna, Bogota, Colombia.

6. LEÓN Carlos. (2002). Cuantificación de los Beneficios de la Lactancia Materna: Reseña de la Evidencia. Washington: Organización Panamericana de la Salud. EEUU

7. COLOMER, Ana (2000). ¿Qué Influye en las Decisiones Sobre la Lactancia? Na Esp Pediatr; 53(2): 167-8. Academia Americana de Pediatría, Argentina

8. GUTIERREZ, FELIPE: (2002), Factores Sociales y Psicológicos de la Lactancia Materna Exclusiva, Guía Practica para Profesionales México.

9. BARRIUSO Luis LM, (2002) Factores Relacionados con el Abandono de la Lactancia Materna. Tomo 1. Madrid: Ed Médica Panamericana SA; 472-483.

10. GUÍA PRACTICA DE LACTANCIA MATERNA, (2001), Educación Para La Lactancia Materna. Calamaco-Cojedes, Venezuela

11.- TEXTO PRACTICO SOBRE LACTANCIA MATERNA (2000) Educación Para La Lactancia Materna. Calamaco-Cojedes.

12.- MERCER RAMONA, TEORÍA DEL ROL MATERNO (1999) Teorías de Enfermería R. L. Wesley (1997): Teorías y modelos de enfermería. McGraw-Hill Interamericana, México.

13.- OLDS, Sally et al: (2000) Enfermería Materno Infantil, (Cuidado integral del Recién Nacido), Interamericana, Quinta edición. México

14.- HELSING, ElizABETH, F. SAVAGE, (1995), Guía Practica Para Una Buena Lactancia (Factores que Inciden en la Practica de la Lactancia Materna), Cuarta edición. México, PAx México

15.- TRATADO DE PUERICULTURA, (1996), (Factores Sociales y Culturales de la Lactancia Materna), Universidad de Carabobo: , tercera edición. Venezuela.

16.- GAMBARA, HILDA. (1.998) Diseño de Investigaciones, Cuaderno de Prácticas. Editorial McGRAW - Hill, Segunda Edición Madrid. 
๑ COPYRIGHT Servicio de Publicaciones - Universidad de Murcia 